\title{
Evaluación de la capacidad de carga de tubos de hormigón reforzado con fibras híbridas
}

\section{Assessment of bearing capacity of hybrid fiber reinforced concrete pipes}

Presentación: 06/10/2020

\section{Doctorando:}

\section{Federico Alejandro González}

Universidad Tecnológica Nacional, Facultad Regional Concepción del Uruguay - Argentina

gonzalezf@frcu.utn.edu.ar

\section{Director/es:}

\section{Viviana Carolina Rougier}

\section{Co-director/es:}

\section{Mario Raúl Escalante}

\section{Resumen}

En las últimas décadas, se ha extendido el uso de un solo tipo de fibras, ya sean de acero o sintéticas, como refuerzo del hormigón en tubos premoldeados. Sin embargo, el hormigón tiene comportamiento a la fractura en múltiples escalas y un tipo de fibra proporciona refuerzo solo en un nivel y dentro de un rango limitado de deformaciones. Por ello, un refuerzo más eficiente podría lograrse utilizando la combinación de fibras de diferentes materiales, geometría y/o características mecánicas. El hormigón reforzado con dos o más tipos de fibras, combinadas racionalmente, se denomina Hormigón Reforzado con Fibras Híbridas (HRFH).

En este trabajo, se presentan los resultados de una campaña experimental en la que se estudió la capacidad de carga de tubos de HRFH (THRFH), sometidos al ensayo de compresión diametral de tres aristas. Los resultados se comparan con los obtenidos para tubos de hormigón con refuerzo tradicional de barras de acero (THA) y tubos de hormigón reforzado con fibras de acero (THRFA), elaborados y ensayados en la misma campaña. Los resultados mostraron que los THRFH alcanzaron una capacidad de carga equivalente a los THA y superior a la de los THRFA.

Palabras clave: fibras híbridas, hormigón reforzado, tubos.

\begin{abstract}
In recent decades, the use of a single type of fiber, either steel or synthetic, as concrete reinforcement in precast pipes. However, concrete has a multi-scale nature of fracture and one type of fiber provide reinforcement only at one level and within a limited range of strains. Therefore, a more efficient reinforcement could be achieved by using the combination of fibers of different materials, geometry and/or mechanical characteristics. Reinforced concrete with two or more types of fibers combined rationally is called hybrid fiber reinforced concrete (HFRC).

In this paper, the results of an experimental campaign are presented in which the bearing capacity of HFRC (HFRCP) pipes was studied. The results are compared with those obtained for concrete pipes with traditional steel bar reinforcement (RCP) and steel fiber reinforced concrete pipes (SFRCP), elaborated and tested in the same campaign. The results showed that the HFRCP reached a load capacity equivalent to RCP and superior to that of SFRCP.
\end{abstract}

Keywords: hybrid fibers, reinforced concrete, pipes. 


\section{Introducción}

El hormigón reforzado con fibra (HRF) se ha utilizado con o sin refuerzo convencional en numerosas aplicaciones en todo el mundo (Yoo et al., 2016), entre ellas, la construcción de nuevos pavimentos, estructuras hidráulicas, losas de puentes, pisos industriales y un gran número de elementos prefabricados, entre los que se encuentran los tubos de drenaje.

En las últimas dos décadas, los tubos de HRF con reemplazo parcial o total del refuerzo tradicional de barras de acero (jaula de acero), se han introducido con éxito en el mercado de tuberías para la construcción de alcantarillados (Mohamed et al., 2015). Una de las principales ventajas del HRF es que las mismas son adicionadas a la hormigonera de igual manera que los otros agregados sin la necesidad de realizar modificaciones significativas en las plantas de premoldeados, es decir, el HRF puede ser colado en los moldes de manera similar al hormigón simple. En cambio, para la fabricación de tubos de hormigón armado se requiere mano de obra calificada para el corte, doblado y montaje de las armaduras formadas por barras de acero, además de la necesidad de disponer de equipamiento adecuado para realizar dichas tareas (Peyvandi et al., 2014) Por lo expresado anteriormente, el uso de HRF en tubos premoldeados conduce a beneficios desde el punto de vista técnico y económico (Lee et al., 2019).

Actualmente, existen normas que regulan la utilización del HRF para la fabricación de tubos, siendo la EN 1916:2002 (2002), "Concrete pipes and fittings, unreinforced, steel fibre and reinforced", la base conceptual de todas ellas y el resto de las normas existentes son adaptaciones de diferentes países (UNE- EN 1916:2008 (2008) en España; ABNT NBR 8890:2020 (2020) en Brasil; ASTM C1765-19 (2019) y ASTM C1818-19 (2019) en EEUU). Sin embargo, dichas normas contemplan la utilización de un solo tipo de fibra como refuerzo, mientras que el uso de la combinación de fibras de diferentes materiales, geometría o propiedades mecánicas en este tipo de elemento ha sido escasamente estudiado (Lee et al., 2019).

El hormigón reforzado con dos o más tipos de fibras combinadas racionalmente se denomina hormigón reforzado con fibras híbridas (HRFH). El principal propósito de usar fibras híbridas es mejorar el control de la fisuración en diferentes zonas del hormigón, a distintas edades y niveles de carga (Mogileswar \& Veni, 2018). Además, dos o más tipos de fibras, combinadas adecuadamente, generan mejoras en las propiedades físicas y mecánicas del material a partir de la acción de cada una de ellas (Banthia \& Nandakumar, 2003), lo que conduce a la mejora de la respuesta estructural del elemento analizado (Banthia et al., 2014; Buratti et al., 2011; Yin et al., 2015; Xu et al., 2017).

En este trabajo se analiza experimentalmente la capacidad de carga de THRFH, de acero y polipropileno, de $600 \mathrm{~mm}$ de diámetro interior sometidos al ensayo de compresión diametral de tres aristas. Los resultados se comparan con los obtenidos para tubos de hormigón armado (THA) Clase II según la norma IRAM 11503 (1986) y con tubos de hormigón reforzados con fibras de acero (THRFA), elaborados y ensayados durante la misma campaña experimental.

\section{Campaña Experimental}

\section{Dosificación del hormigón.}

Los especímenes ensayados se elaboraron en una empresa de premoldeados de la región. Para la presente investigación, se adoptó la misma proporción de la mezcla (ver Tabla 1) que utiliza la empresa para elaborar los tubos que comercializa. La mezcla de hormigón tiene una consistencia muy seca debido a que, durante la producción, el tubo se desmolda casi inmediatamente después del llenado, por ello se requiere una mezcla con gran cohesión y baja relación agua-cemento.

\begin{tabular}{|c|c|c|c|}
\hline Agua & Cemento & Arena & Grava \\
\hline 80 & 300 & 980 & 1020 \\
\hline
\end{tabular}

Tabla 1: dosificación teórica del hormigón simple en $\mathrm{kg} / \mathrm{m}^{3}$.

Se utilizaron agregados de la zona, canto rodado de tamaño máximo $1 \frac{1}{2}$ " y arena fina de río $(M F=2,52)$. Se realizaron ensayos de compresión sobre probetas cilíndricas obteniéndose una resistencia media de 38MPa. Las fibras fueron agregadas manualmente a la mezcla, arrojándolas dentro de la mezcladora con la precaución de distribuirlas uniformemente en toda la superficie, a fin de evitar la formación de erizos.

\section{Fibras Utilizadas.}

Para el refuerzo de los tubos se utilizaron macrofibras de acero y de polipropileno disponibles en el mercado nacional. Las macrofibras MACRONITA ${ }^{\circledR}$ son fibras sintéticas recortadas de materiales poliméricos. Este tipo de fibras son químicamente inertes, tienen estabilidad volumétrica, un bajo módulo de elasticidad y bajo punto de fusión. Las 
fibras de acero (WIRAND ${ }^{\circledR}$ FF1) se fabrican con alambre de acero de bajo carbono y de alta resistencia a la tracción $1200 \mathrm{MPa}$. Al contrario de las fibras de polipropileno, las fibras de acero tienen un alto módulo de elasticidad y son susceptibles a la corrosión. En la Tabla 2, se presentan las características geométricas de las fibras utilizadas, algunas de sus propiedades más importantes y se pueden observar sus formas.

\begin{tabular}{|l|c|c|c|c|c|}
\hline \multirow{2}{*}{ Material } & Longitud & Diámetro & Densidad & Módulo & \multirow{2}{*}{ Formas } \\
\cline { 2 - 5 } & $\mathrm{mm}$ & $\mathrm{mm}$ & $\mathrm{kg} / \mathrm{m}^{3}$ & $\mathrm{GPa}$ & \\
\hline Acero & 50 & 1,00 & 7840 & 210 & \\
\hline Polipropileno & 50 & 0,60 & 920 & 6 & \\
\hline
\end{tabular}

Tabla 2: características de las fibras empleadas.

\section{Elaboración de Ios THA, THRFA y THRFH.}

Para la fabricación de los tubos se utilizó una mezcladora tipo planetaria de eje vertical y el tiempo de mezclado, una vez agregadas las fibras, fue de 2 minutos aproximadamente. Luego, el hormigón fue transportado desde la hormigonera hasta el molde por medio de una cinta transportadora. El molde estaba compuesto por tres partes: una parte externa, una parte interna y un anillo que sirvió como base para el tubo durante el moldeado. La parte externa era una camisa de acero cilíndrica desmontable y la parte interna era el núcleo vibratorio, que fue el responsable de la vibración del hormigón mientras era colado en el molde. Luego del llenado y la compactación del tubo, el núcleo vibratorio fue izado y el tubo fue transportado hasta el lugar de curado, en donde fue desmontada la camisa externa para continuar con el proceso de elaboración, reutilizando dicha camisa y el núcleo vibratorio. En la Figura 1 se muestran algunas partes del proceso de elaboración de los tubos: moldeo, transporte hasta el lugar de almacenamiento y desmolde.
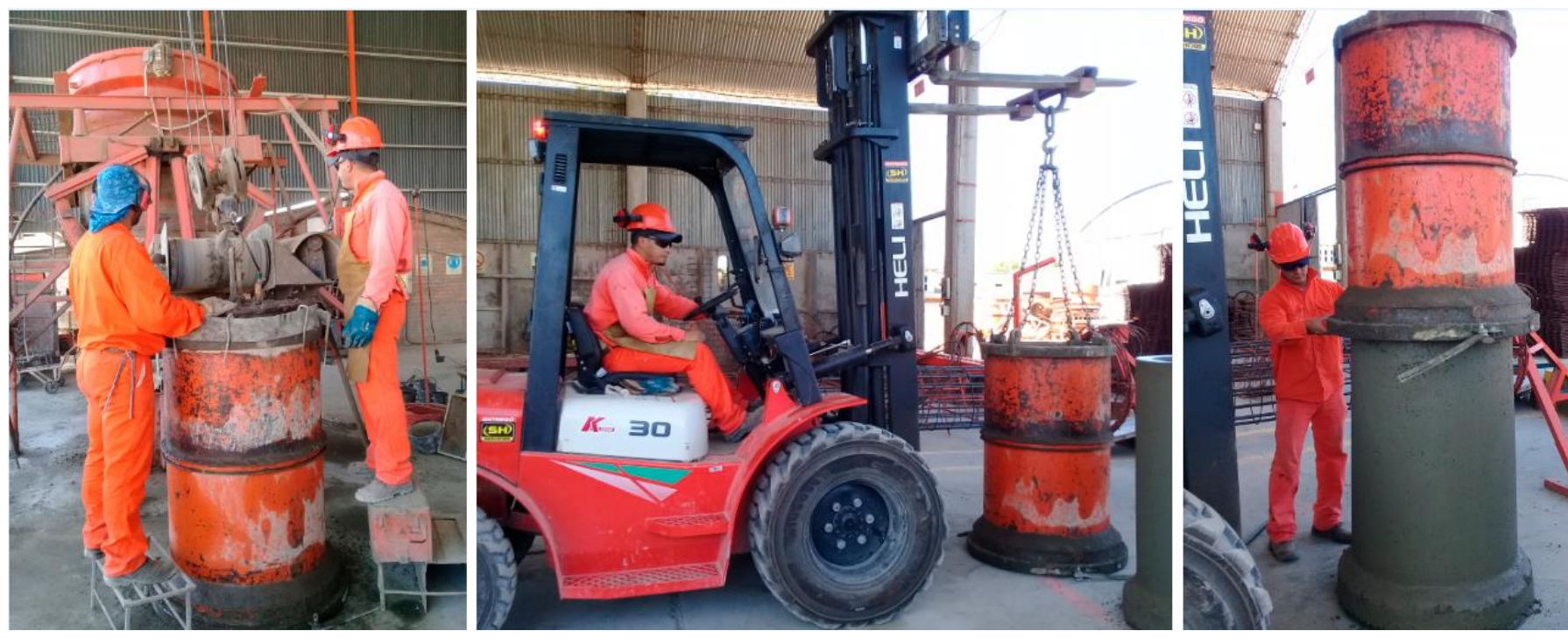

Figura 1: moldeo (izquierda); transporte hasta el lugar de acopio (centro); desmolde (derecha).

\section{Características de los especímenes.}

Fueron elaborados para su estudio tubos de $600 \mathrm{~mm}$ de diámetro interior, $1000 \mathrm{~mm}$ de longitud y un espesor de pared de $75 \mathrm{~mm}$. Se adoptaron estas dimensiones debido a que son las mínimas establecidas por la norma IRAM 11503 (1986) para tubos Clase II.

En la Tabla 3 se muestra la cantidad y denominación de los especímenes a ensayar agrupados de acuerdo a sus características de refuerzo.

El grupo THA corresponde a los tubos reforzados con armadura tradicional de barras de acero. La característica de la misma es establecida en la norma IRAM 11503 (1986) para tubos de Clase II y está formada por 7 barras de acero de $6 \mathrm{~mm}$ de diámetro en sentido longitudinal y una cuantía de $1,5 \mathrm{~cm}^{2} / \mathrm{m}$ en sentido transversal.

Los grupos I y II corresponden a los tubos reforzados con fibras de acero, mientras que los grupos III y IV a los reforzados con fibras híbridas de acero y polipropileno.

En cuanto a las dosis de fibras usadas, se adoptaron $20 \mathrm{~kg} / \mathrm{m}^{3}$ y $25 \mathrm{~kg} / \mathrm{m}^{3}$ de FA para los THRFA debido a que otros autores han demostrado la eficiencia del refuerzo en estos tenores (Banthia et al., 2014; Park et al., 2015; Lee et al., 2019). Por otra parte, se adoptaron dosis de $0,5 \mathrm{~kg} / \mathrm{m}^{3}$ y $1 \mathrm{~kg} / \mathrm{m}^{3} \mathrm{de}$ FPP combinadas con $20 \mathrm{~kg} / \mathrm{m}^{3}$ de FA. Cuando las fibras de PPF se encuentran combinadas con otras, se recomiendan fracciones de bajo volumen comprendidas entre 
$0,05 \%$ y 0,2 \% con el fin de evitar la aparición de erizos localizados y asegurar la distribución uniforme de las fibras (Li et al., 2018).

Como se observa en la Tabla 3, el Grupo II y III tienen adicionado el mismo volumen de fibras de refuerzo, mientras que para el grupo IV este volumen es incrementado de $0,30 \%$ a $0,35 \%$ con la adición de $1 \mathrm{~kg} / \mathrm{m}^{3} \mathrm{de} P P F$.

\begin{tabular}{|c|c|c|c|c|c|}
\hline \multirow{2}{*}{ Grupo } & \multirow{2}{*}{ Denominación } & FA & FPP & $\begin{array}{c}\text { Volumen } \\
\text { de fibras }\end{array}$ & Cantidad \\
\cline { 3 - 6 } & & $\mathrm{kg} / \mathrm{m}^{3}$ & $\mathrm{~kg} / \mathrm{m}^{3}$ & $\%$ & Unid. \\
\hline THA & THA-0/0 & ---- & ---- & 0 & 4 \\
\hline I & THRFA-20/0 & 20 & 0 & 0,25 & 4 \\
\hline II & THRFA-25/0 & 25 & 0 & 0,30 & 4 \\
\hline III & THRFH-20/0,5 & 20 & 0,5 & 0,30 & 4 \\
\hline IV & THRFH-20/1,0 & 20 & 1,0 & 0,35 & 4 \\
\hline
\end{tabular}

Tabla 3: características de refuerzo y cantidad de tubos ensayados.

\section{Ensayo de compresión diametral.}

Todos los tubos elaborados fueron ensayados a compresión diametral, de acuerdo a los procedimientos que establece la norma IRAM 11503 (1986) para la determinación de la carga de rotura $\left(\mathrm{F}_{\mathrm{R}}\right)$. El ensayo de compresión diametral es también denominado de "tres aristas" debido a que el tubo es apoyado sobre dos largueros de caucho rígido y la carga se aplica en el eje generatriz superior, a través de un perfil metálico que la distribuye uniformemente. La carga aplicada debe ser cuasi-estática monótona creciente y para su aplicación se usó una prensa con un sistema hidráulico con capacidad para desarrollar una carga vertical nominal máxima de $140 \mathrm{kN}$. Los valores de carga se registraron a través de la utilización de una celda de carga de 10 Ton. En la Figura 2 se muestra la colocación del tubo en el marco de carga y el tubo luego de su rotura.
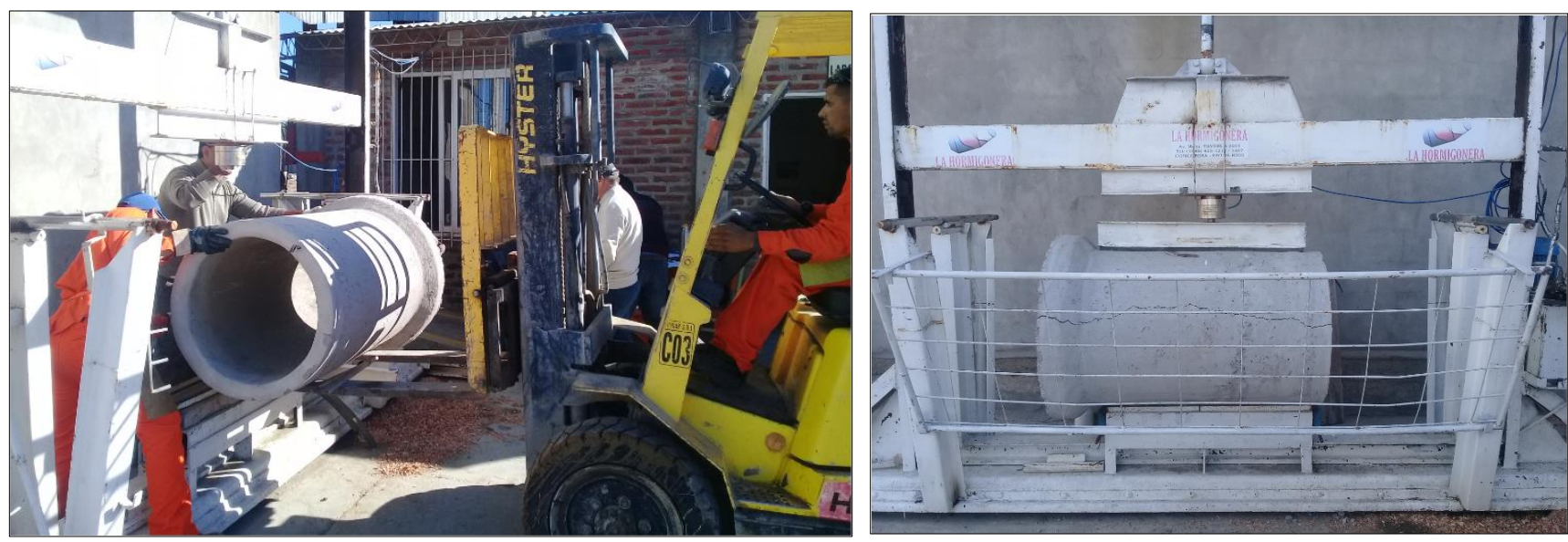

Figura 2: Colocación del especímen en el marco de carga (izquierda); Tubo luego del ensayo (derecha).

\section{Resultados}

En la Tabla 4 se observan las cargas de rotura $\left(F_{R}\right)$ de cada espécimen, las cargas de rotura promedio ( $\left.F_{R M}\right)$ por grupo de especímenes y el coeficiente de variación de la $\mathrm{F}_{\mathrm{RM}}$ de cada grupo, respecto a la del grupo de control (THA).

La norma IRAM 11503 (1986) establece una carga mínima de rotura igual a $45 \mathrm{kN}$ para la Clase II. Se observa que los THRFH, con ambas dosificaciones, alcanzaron y superaron la carga mínima de rotura mencionada. Además, los THRFH-20/0,5 y los THRFH-20/1,0 alcanzaron una $\mathrm{F}_{\text {RM }}$ equivalente a los THA, siendo su COV igual a 1,39 \% y -2.77 \%, respectivamente.

Por el contrario, los THRFA-20/0 y los THRFA-25/0 no alcanzaron el valor mínimo de carga de rotura para la Clase II (IRAM 11503, 1986) siendo inferiores en 10,4 \% y 15,5 \%, respectivamente. Además, los THRFA con ambas dosificaciones mostraron una capacidad de carga significativamente inferior a los THA con un COV mayor al $20 \%$. 


\begin{tabular}{|c|c|c|c|c|c|}
\hline & FA & FPP & $F_{R}$ & $\mathbf{F}_{\mathrm{RM}}$ & COV \\
\hline Denominacion & $\mathrm{Kg} / \mathrm{m}^{3}$ & $\mathrm{Kg} / \mathrm{m}^{3}$ & $\mathrm{KN}$ & $\mathrm{KN}$ & $\%$ \\
\hline 1-THA & - & - & 51,0 & \multirow{4}{*}{50,5} & \multirow{4}{*}{0} \\
\hline 2-THA & - & - & 48,0 & & \\
\hline 3-THA & - & - & 55,0 & & \\
\hline 4-THA & - & - & 48,0 & & \\
\hline 1-THRFA-20/0 & 20 & - & 35,0 & \multirow{4}{*}{40,3} & \multirow{4}{*}{$-20,19$} \\
\hline 2-THRFA-20/0 & 20 & - & 40,0 & & \\
\hline 3-THRFA-20/0 & 20 & - & 43,0 & & \\
\hline 4-THRFA-20/0 & 20 & - & 43,0 & & \\
\hline 1-THRFA-25/0 & 25 & - & 36,0 & \multirow{4}{*}{38,0} & \multirow{4}{*}{$-24,75$} \\
\hline 2-THRFA-25/0 & 25 & - & 42,0 & & \\
\hline 3-THRFA-25/0 & 25 & - & 36,0 & & \\
\hline 4-THRFA-25/0 & 25 & - & 38,0 & & \\
\hline 1-THRFH-20/0,5 & 20 & 0,5 & 53,0 & \multirow{4}{*}{51,2} & \multirow{4}{*}{1,39} \\
\hline 2-THRFH-20/0,5 & 20 & 0,5 & 45,0 & & \\
\hline 3-THRFH-20/0,5 & 20 & 0,5 & 54,0 & & \\
\hline 4-THRFH-20/0,5 & 20 & 0,5 & 52,7 & & \\
\hline 1-THRFH-20/1,0 & 20 & 1,0 & 47,0 & \multirow{4}{*}{49,1} & \multirow{4}{*}{$-2,77$} \\
\hline 2-THRFH-20/1,0 & 20 & 1,0 & 46,0 & & \\
\hline 3-THRFH-20/1,0 & 20 & 1,0 & 51,5 & & \\
\hline 4-THRFH-20/1,0 & 20 & 1,0 & 52,0 & & \\
\hline
\end{tabular}

Tabla 4: cargas de rotura por especímen $\left(F_{R}\right)$, carga de rotura promedio por grupo $\left(F_{R M}\right)$ y COV.

La mayor $\mathrm{F}_{\mathrm{RM}}$ fue alcanzada por los THRFH-20/0,5 y superó los valores promedio de los THRFA-20/0 y los THRFA$25 / 0$ en $27 \%$ y $34,7 \%$, respectivamente. Basándonos en estos promedios, podemos observar que la resistencia a la rotura de los tubos se incrementó significativamente con la utilización de la combinación de fibras como refuerzo, en comparación con aquellos especímenes que fueron reforzados con la FA solamente. Las fibras, en general, forman una red tridimensional en la masa del hormigón que le impide que segregar y fluir, por lo cual, la adición de un pequeño porcentaje de FPP le otorga mayor cohesión a la mezcla. Sin embargo, superado cierto volumen límite de fibras, la mayor viscosidad de la mezcla traería problemas de compactación que afectarían el comportamiento de la pieza durante el ensayo. Lo expresado anteriormente se puede observar al comparar los resultados obtenidos para los THRFH-20/0,5 con un volumen de fibras de 0,30 \% y los THRFH-20/1,0 con un volumen de 0,35\%, donde la capacidad de carga no se incrementa al aumentar el volumen de fibras, sino por el contrario, la misma baja en un 4,26\% aproximadamente. Además, una observación similar puede realizarse al comparar los THRFA-20/0 con 0,25 \% y los THRFA-25/0 con 0,30 \% de volumen de FA, donde la capacidad de carga disminuyó en $6 \%$. A pesar de lo expuesto anteriormente sobre el volumen fibras,

\section{Conclusiones}

En el presente trabajo se evaluó la capacidad de carga de THRFH a través del ensayo de compresión diametral de tres aristas, y los resultados fueron comparados con los obtenidos para THA y THRFA. Para ello, se elaboraron y ensayaron hasta la rotura 8 THRFA, 8 THRFH y 4THA de $600 \mathrm{~mm}$ de diámetro interno. Se adoptaron dos dosificaciones distintas para los THRFH y dos para los THRFA. Del análisis basado en los resultados obtenidos, se puede concluir lo siguiente:

- La capacidad de carga de los tubos se incrementó significativamente al usar como refuerzo del hormigón a la combinación de FA y FPP, en ambas dosis utilizadas.

- El aumento en el volumen de FPP en los THRFH produjo una leve disminución de la capacidad de carga.

- Para un mismo volumen de fibras, la carga de rotura en promedio de los THRFH superó a la de los THRFA.

- Los THRFH alcanzaron una carga de rotura promedio equivalente a la de los THA Clase II. 


\section{Referencias}

ASTM C1765-19 (2019). Standard specification for steel fiber reinforced concrete culvert, storm drain, and sewer pipe. Standard, American Society for Testing and Materials International, West Conshohocken, PA.

ASTM C1818-19 (2019). Standard specification for synthetic fiber reinforced concrete culvert, storm drain, and sewer pipe. Standard, American Society for Testing and Materials International, West Conshohocken, PA.

Banthia, N., \& Nandakumar, N. (2003). Crack growth resistance of hybrid fiber reinforced cement composites. Cement and Concrete Composites, 25(1), 3-9.

Banthia, N., Majdzadeh, F., Wu, J., \& Bindiganavile, V. (2014). Fiber synergy in Hybrid Fiber Reinforced Concrete (HyFRC) in flexure and direct shear. Cement and Concrete Composites, 48, 91-97.

Buratti, N., Mazzotti, C., \& Savoia, M. (2011). Post-cracking behaviour of steel and macro-synthetic fibre-reinforced concretes. Construction and building materials, 25(5), 2713-2722.

EN 1916:2002/AC:2008 (2002). Concrete pipes and fittings, unreinforced, steel fibre and reinforced. Standard, European Committee for Standardization.

IRAM 11503 (1986). Caños de hormigón armado no pretensado. Destinados a la conducción de líquidos sin presión. Instituto Argentino de Racionalización de Materiales.

Lee, S., Park, Y., \& Abolmaali, A. (2019). Investigation of Flexural Toughness for Steel-and-Synthetic-Fiber-Reinforced Concrete Pipes. Structures, 19, 203-211.

Li, B., Chi, Y., Xu, L., Shi, Y., \& Li, C. (2018). Experimental investigation on the flexural behavior of steel-polypropylene hybrid fiber reinforced concrete. Construction and Building Materials, 191, 80-94.

Mogileswar, K., \& Veni, B. (2018). Experimental Investigation on Hybrid Fiber Reinforced Concrete (M25) and its Effects on properties of Strength and Durability. International Research Journal of Engineering and Technology, 5. Mohamed, N., Soliman, A. M., \& Nehdi, M. L. (2015). Mechanical performance of full-scale precast steel fibre-reinforced concrete pipes. Engineering Structures, 84, 287-299.

NBR ABNT 8890:2020 (2020). Tubo de concreto de seção circular para água pluvial e esgoto sanitário - requisitos e métodos de ensaios. Standard, Associaçao Brasileira de Normas Técnicas, Av. Treze de Maio, RJ.

Park, Y., Abolmaali, A., Mohammadagha, M., \& Lee, S. (2015). Structural performance of dry-cast rubberized concrete pipes with steel and synthetic fibers. Construction and Building Materials, 77, 218-226.

Peyvandi, A., Soroushian, P., \& Jahangirnejad, S. (2014). Structural design methodologies for concrete pipes with steel and synthetic fiber reinforcement. ACI Structural Journal, 111(1), 83.

Xu, L., Deng, F., \& Chi, Y. (2017). Nano-mechanical behavior of the interfacial transition zone between steelpolypropylene fiber and cement paste. Construction and Building Materials, 145, 619-638.

Yin, S., Tuladhar, R., Shi, F., Combe, M., Collister, T., \& Sivakugan, N. (2015). Use of macro plastic fibres in concrete: A review. Construction and Building Materials, 93, 180-188.

Yoo, D. Y., \& Yoon, Y. S. (2016). A review on structural behavior, design, and application of ultra-high-performance fiber-reinforced concrete. International Journal of Concrete Structures and Materials, 10(2), 125-142. 